\title{
Review
}

\section{Neural Crest-derived Cells in the Oral and Maxillofacial Regions of Adult Mice: Isolation and Application for Regenerative Medicine}

\author{
Tetsuo SuZawA ${ }^{* 1)}$, Hiroshi YoshidA ${ }^{1,2)}$, Masakatsu ITose ${ }^{1,3)}$, \\ Reiko Takimoto ${ }^{1,3)}$, Emi Saito ${ }^{1,4)}$, Dai Suzuki ${ }^{1)}$, \\ Katsunori InAGAKI ${ }^{4)}$, Tatsuo ShIROTA ${ }^{3)}$, Koutaro MAKI ${ }^{2)}$ \\ and Ryutaro KAMIJO ${ }^{1)}$
}

\begin{abstract}
Neural crest cells emerge from the dorsal region of the fusing neural tube in vertebrate embryos, then migrate throughout tissues to differentiate into various cell types, including osteoblasts. In adults, subsets of neural crest-derived cells (NCDCs) reside as stem cells and are considered to be useful cell sources for regenerative medicine. Previous studies have suggested that these NCDC subsets persist into adulthood in mammals, especially those cells within the craniofacial compartments. Recently, our group found that NCDCs were scattered throughout tissues of the palate, gingiva, tongue, hair follicle, submandibular glands, and buccal mucosa of adult mice. NCDCs from the buccal mucosa can also form neurosphere-like structures that have the capability to differentiate into osteoblasts in the presence of bone morphogenetic protein-2. In addition, NCDCs in adults have characteristic gene expression profiles, especially their cell surface molecules. Thus, cell sorting using several specific cell surface molecules has been proposed as a useful method for isolating NCDCs with high purity. Together, these results suggest that NCDCs reside in various adult oral and maxillofacial regions, and possess the potential to differentiate into osteoblastic cells, indicating that these cells in adults may be a useful source for bone regeneration strategies. In this review, we discuss the distribution, isolation and osteoblastic differentiation potential of NCDCs isolated from various adult tissue sources in the oral and maxillofacial regions.
\end{abstract}

Key words : neural crest-derived cells, adult, cellular differentiation, osteoblasts, oral and maxillofacial regions

\section{Introduction}

The neural crest $(\mathrm{NC})$ is a transitory structure of the vertebrate embryo that arises from a region at the border of the neural plate, between the neural plate and the adjacent non-neural

1) Department of Biochemistry, Showa University School of Dentistry, 1-5-8 Hatanodai, Shinagawa, Tokyo 142-8666, Japan.

2) Department of Orthodontics, Showa University, School of Dentistry.

3) Department of Oral and Maxillofacial Surgery, Showa University, School of Dentistry.

4) Department of Orthopedic Surgery, Showa University, School of Medicine.

* To whom corresponding should be addressed. 

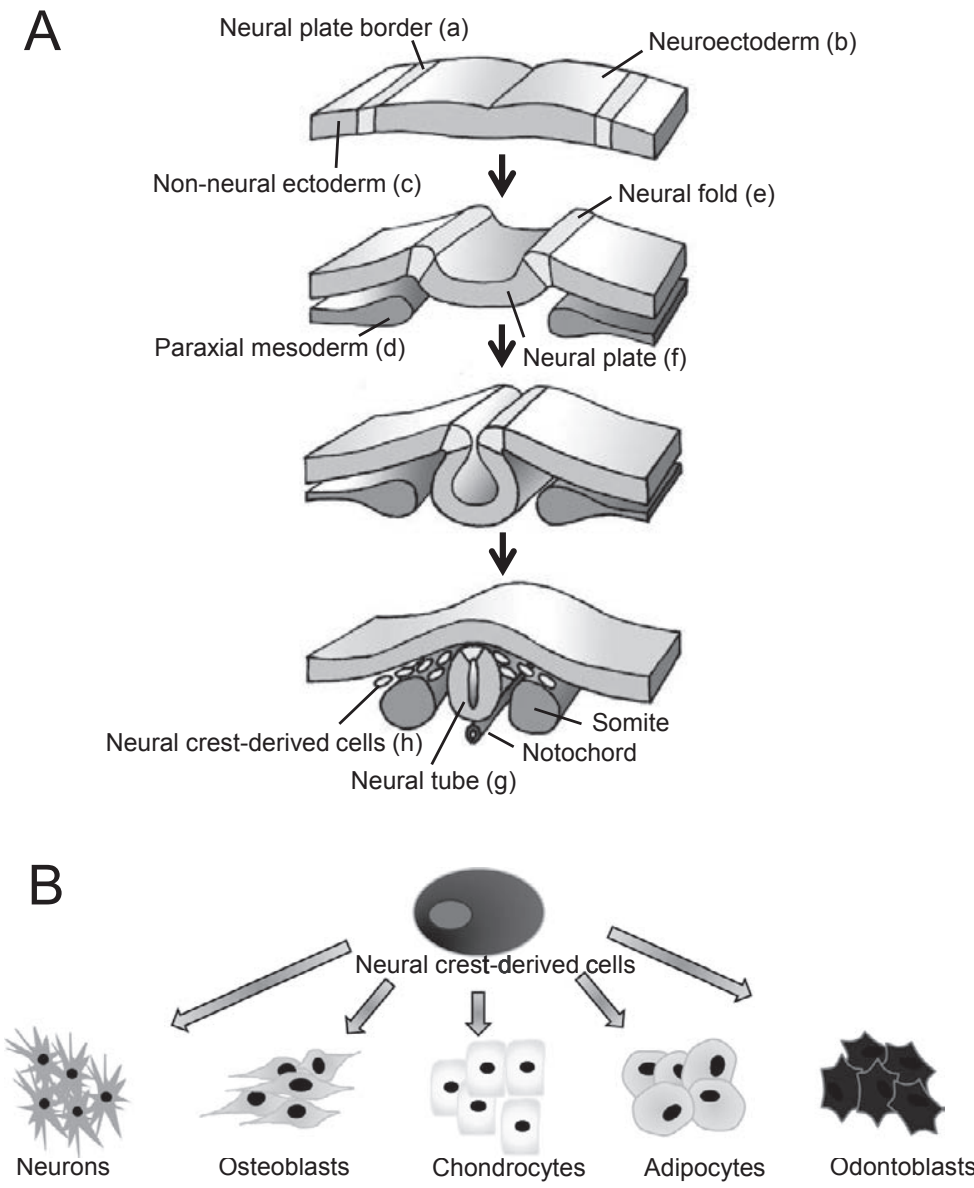

Fig. 1 .

(A) The neural plate border (a) is induced by signaling between the neuroectoderm (b) and nonneural ectoderm (c), as well as from the underlying paraxial mesoderm (d). During neurulation, the neural plate borders elevate [neural folds (e)], causing the neural plate (f) to roll into a neural tube $(\mathrm{g})$. Neural crest-derived cells (h) delaminate from the neural folds or dorsal neural tube. Source: Adapted from Gammill and Bronner-Fraser $(2003)^{1)}$ with permission from Nature Publishing Group. Copyright ${ }^{\oplus}$ 2003, Macmillan Publishers Limited. All rights reserved.

(B) Neural crest-derived cell derivatives. Neural crest-derived cells originate from the neural tube and show pluripotency. They migrate extensively, and give rise to a vast array of cell types and organs. In adults, these cells can undergo self-renewal as well as multi-lineage differentiation.

ectoderm. This structure remains at the neural plate border during neurulation and transformation of the neural plate into rising neural folds ${ }^{1)}$. After neural tube closure, the $\mathrm{NC}$ comes to reside in a domain of the dorsal neural tube (Fig. 1A). Thereafter, NC cells originate from the dorsal aspect of the neural tube ${ }^{2)}$, undergo an epithelium to mesenchyme transition ${ }^{3)}$, and become delaminated from the neural tube as NC-derived cells (NCDCs). During vertebrate development, NCDCs migrate in a ventro-lateral manner ${ }^{4)}$ and populate the branchial arches, while they extensively contribute to the formation of mesenchymal structures in the head during migration ${ }^{5-7)}$. Furthermore, NCDCs also have a self-renewal capability and give rise to a multitude of cell types that include neurons, glial cells, myofibroblasts, melanocytes, adipocytes, chondrocytes, osteoblasts, and odontoblasts (Fig. 1B) ${ }^{8-10)}$. 
Recent studies have suggested the continuous survival of these highly pluripotent cells into adulthood, along with their high regenerative potential, and this has been demonstrated in a variety of mammalian craniofacial tissues ${ }^{11-13)}$. Interestingly, some NCDCs are maintained in an undifferentiated condition throughout the life of the animal ${ }^{14,15)}$, while those obtained from the oral and olfactory mucosa, which are easily accessible in the oral and maxillofacial regions, can be cultured ${ }^{11-13,16)}$ and may represent a useful cell source for regeneration of those regions.

In this review, we describe findings regarding the distribution, isolation, and characterization of NCDCs in adult organisms. Moreover, we discuss their specific properties and potential application for cell-based tissue repair strategies.

\section{Distribution of neural crest-derived cells in the oral and maxillofacial regions of adult mice}

Various investigators have reported the precise characteristics of NCDCs, including generation, mobilization, and differentiation during embryogenesis ${ }^{8-13)}$. To analyze the precise distribution and characteristics of NCDCs in adult oral tissues, an established line of double transgenic (P0-Cre/CAG-CAT-EGFP) mice (P0 mice) was utilized, in which NCDCs express green fluorescent protein (GFP) throughout the life of the animal ${ }^{17-20)}$. $P 0$, originally reported as a Schwann cell-specific myelin protein, was previously shown to be transiently activated in migrating NC cells isolated from early chick embryos ${ }^{21)}$. Wnt1 is also expressed specifically in the dorsal neuroepithelium, which includes a pre-migratory population of $\mathrm{NC}$ cells in mice ${ }^{22)}$. Although $P 0$ and Wnt1 are the most reliable markers of NCDCs in embryos, their expressions become completely silenced prior to birth, though recently genetic marking using Cre-recombinase has been applied for long-term tracing of NCDCs in P0-Cre and Wnt1-Cre mice ${ }^{8,17-20)}$. Using such a technique, we observed the distribution of GFP-positive cells, which were considered to have a NC origin, in the buccal mucosa, palate, gingiva, and tongue samples of adult P0 mice (Fig. 2A, A') ${ }^{23}$. Furthermore, Widera et al recently reported the presence of nestin-positive NC-related stem cells within Meissner corpuscles in hard palate tissues of adult rats, and also noted that human palatal cells expressed high levels of the stem cell markers, CD133 and nestin ${ }^{24}$. In agreement with those findings, we observed GFP-positive regions in the palates of adult P0 mice, which also appeared on the surface of the entire palate in a manner similar to the buccal mucosa (Fig. 2B, B'). GFP-positive regions, noted as green dots, were also seen on the surface of the tongue. In addition, Liu et al noted contributions of NCDCs to both tongue mesenchyme and epithelium development in 2 independent mouse lines, Wnt1-Cre and P0-Cre; both express Cre-recombinase in an NC-specific manner ${ }^{25)}$. They also reported that NCDCs are distributed within the lingual epithelium and mesenchyme in close association with embryonic taste papillae formation. The GFP-positive cells found in the tongue in that study may be components of taste papillae and taste buds, though additional analysis is necessary to determine the types of cells in the tongue that are positive for $\mathrm{GFP}^{31)}$.

A few studies have noted the existence of NCDCs in embryonic submandibular glands $(\mathrm{SMGs})^{8,26)}$. It is known that embryonic mesenchymal tissues in SMGs are derived from cranial NC cells and we have demonstrated the existence of GFP-positive cells in SMGs of adult P0 

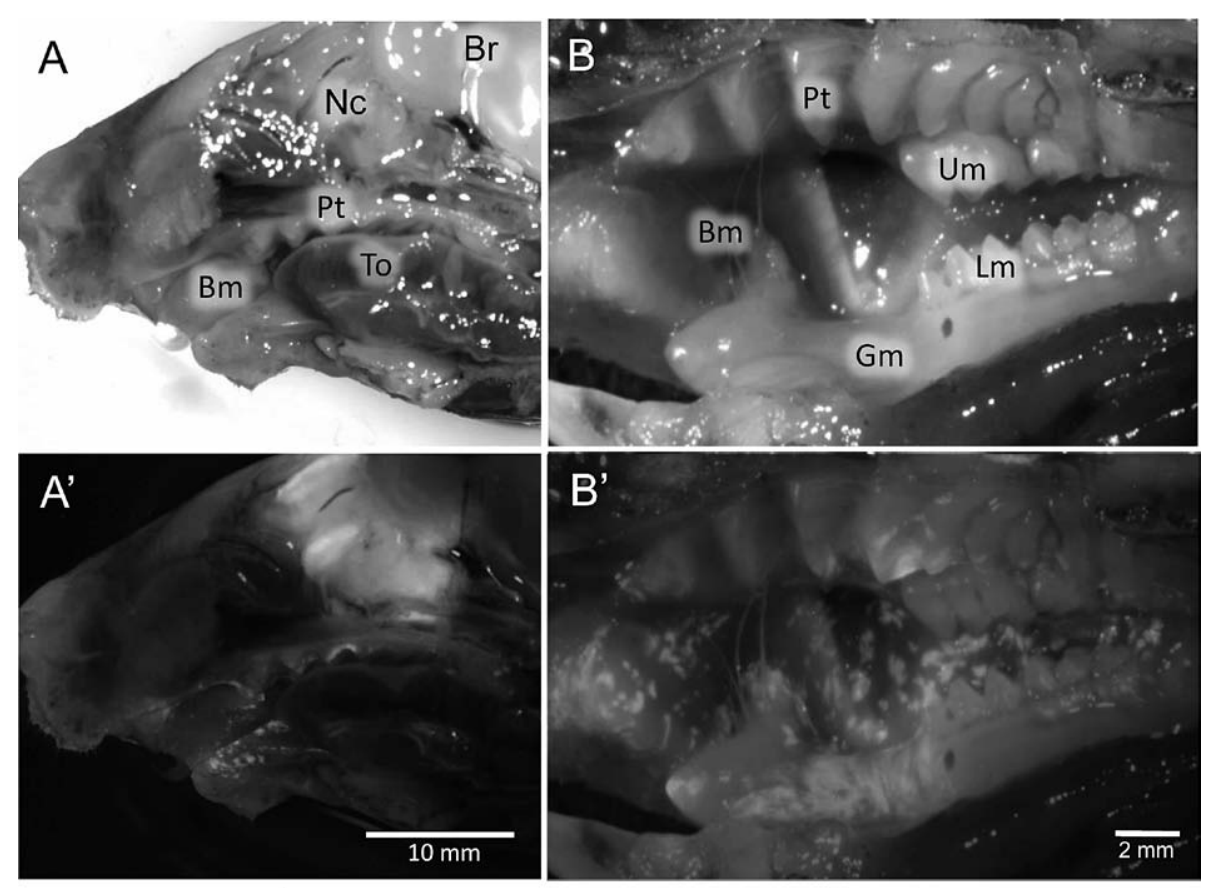

Sagittal section

\section{Oral cavity}

Fig. 2. Distribution of green fluorescent protein-positive cells in adult buccal mucosa, palate, gingiva, and tongue tissues $P 0$-Cre/CAG-CAT-EGFP mice. (A) Mid-sagittal section through the skull examined by florescence stereomicroscopy. (B) Representative appearance of sagittal sections obtained from the oral cavity of an adult mouse. Top panels (A, B) show corresponding bright-field images and bottom panels (A', B') show corresponding fluorescence micrograph images. $\mathrm{Br}$, brain; $\mathrm{Pt}$, palate; $\mathrm{Bm}$, buccal mucosa; $\mathrm{Gm}$, gingival mucosa; To, tongue; Um, upper molars; Lm, lower molars. (A, A') Scale bar $=10 \mathrm{~mm} . \quad\left(B, B^{\prime}\right)$ Scale bar $=2 \mathrm{~mm}$. Source: Adapted from Ono et al $(2015)^{23)}$ with permission from Elsevier. Copyright ${ }^{\circ}$ 2015, Elsevier Inc. All rights reserved.

mice, which were shown as tiny bright islands in the overall SMG samples ${ }^{27)}$. Another study that utilized Wnt1-Cre/ROSA26 transgenic mice showed that embryonic SMG mesenchyme is derived from cranial $\mathrm{NCDCs}^{26)}$, although those NCDCs were restricted to mesenchymal cells in the SMGs and were not found in the epithelium. Platelet-derived growth factor receptor $\alpha$ (Pdgfra) and platelet-derived growth factor receptor $\beta(P d g f r b)$ were also found to be expressed in mesenchymal cells in the SMGs, while GFP-positive cells were observed in the hair follicle bulge, which contains adult stem cells, and the dermal papilla, which retains stem cell-like properties.

\section{Clinical potential of adult neural crest-derived cells}

The multipotent capacity of NCDCs is a characteristic that indicates their potential for use in regenerative medicine. In general, NCDCs are pluripotent in the early stage of embryonic development, after which their potency becomes more restricted following migration from their niche between the ectoderm and neural tube. However, in recent years, several studies have revealed the persistence of NCDCs in adult mammals. Epidermal NC stem cells transplanted into a contused spinal cord caused improvement in sensory connectivity and substantial recovery of touch 

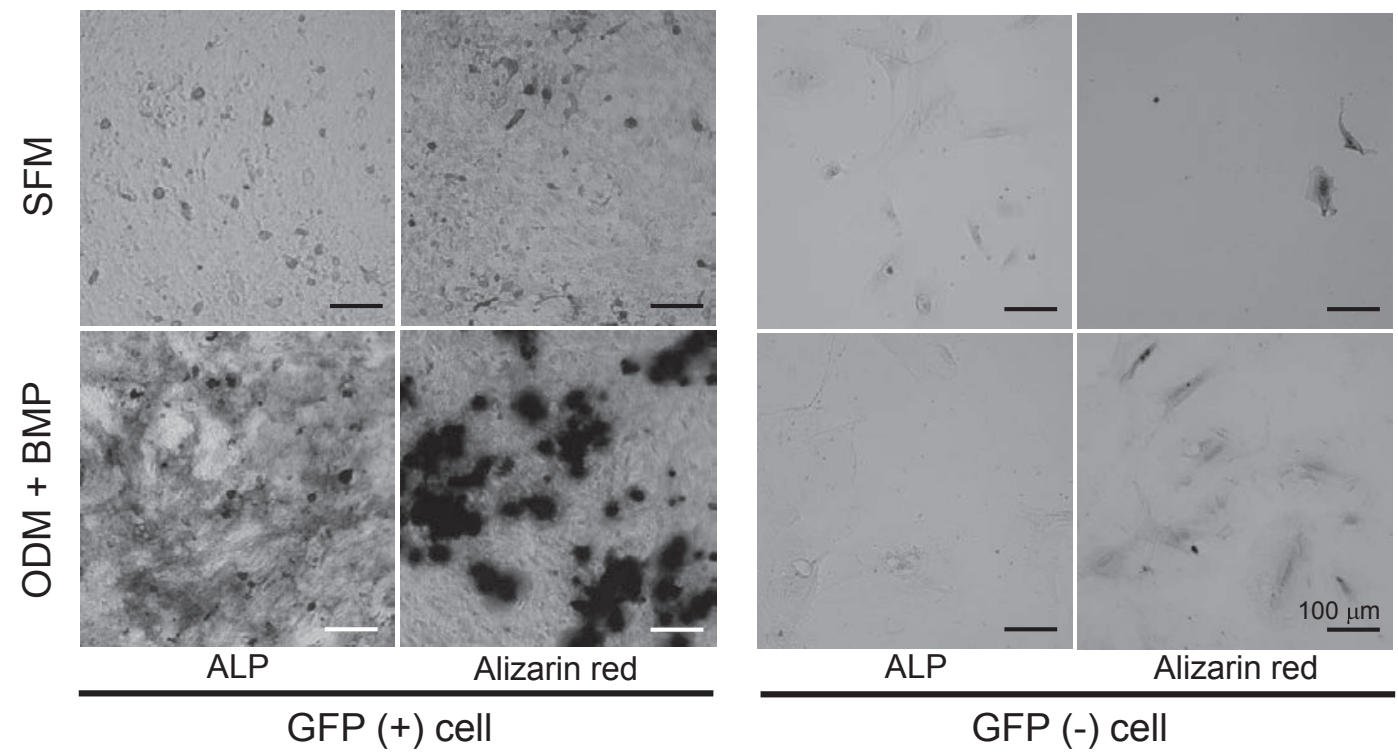

Fig. 3. Osteogenic differentiation of green fluorescent protein (GFP)-positive neural crest-derived cells from the lamina propria of the buccal mucosa of adult P0-Cre/CAG-CAT-EGFP mice. Cells were maintained in culture for 14 days after seeding, then subjected to alkaline phosphatase staining (ALP) and alizarin red staining for calcium deposition (alizarin red). GFP-positive $[$ GFP $(+)]$ and -negative $[$ GFP $(-)]$ cells were cultured in osteoblastic differentiation medium (ODM: Minimum Essential Medium with 10\% fetal bovine serum containing $10 \mathrm{mM} \beta$-glycerol phosphate, $0.5 \mathrm{mM}$ ascorbic acid, and $10^{-8} \mathrm{M}$ dexamethasone) with $200 \mathrm{ng} / \mathrm{ml}$ bone morphogenetic protein-2, or sphere-forming medium (SFM: Dulbecco's Modified Eagle Medium/Nutrient Mixture F-12, supplemented with $20 \mathrm{ng} / \mathrm{ml}$ epidermal growth factor, $20 \mathrm{ng} / \mathrm{ml}$ basic fibroblast growth factor, B27 supplement). Scale bar $=100 \mu \mathrm{m}$. Source: Reproduced from Ono et al $(2015)^{23)}$ with permission from Elsevier. Copyright ${ }^{\odot}$ 2015, Elsevier Inc. All rights reserved.

perception $^{28)}$. Also, the potential of enteric neural cell therapy for gastrointestinal disorders by transplantation of mouse enteric NC cells into ganglionic and aganglionic mouse gut areas has been revealed in vivo. Transplanted enteric NC cells engrafted into ganglionic and aganglionic gut areas showed appropriate spreading and functional integration ${ }^{29)}$. Gingivae in the oral and maxillofacial regions were found to contain both NCDCs and mesoderm-derived mesenchymal stem cells (MSCs), with nearly all of the gingival MSCs shown to be derived from cranial NC cells $^{24)}$. Also, NC cells derived from gingival MSCs showed an elevated capacity to differentiate into chondrocytes and osteoblasts ${ }^{30)}$. In our study, NCDCs were isolated from the lamina propria of the buccal mucosa, and then cultured in sphere-forming and osteoblastic differentiation media. Following isolation of GFP-positive and -negative cells using flow cytometry with a cell sorter, $10 \%-15 \%$ of the buccal mucosa lamina propria cells were found to be positive for $\mathrm{GFP}^{23)}$, When isolated from the lamina propria of the buccal mucosa and cultured, GFP-positive cells formed spheres, a stem cell phenotype, and produced alkaline phosphatase (ALP), a marker enzyme of the early stage of osteoblastic differentiation (Fig. 3). In contrast, GFP-negative cells did not express ALP. Furthermore, mineralization was observed in osteoblastic differentiated GFP-positive cell cultures. Lipid droplets were also noted in adipogenic-stimulated cultures utilizing Oil Red O staining, suggesting that oral NCDCs are multipotent and capable of generating 
osteoblasts and adipocytes ${ }^{23)}$. Consistent with our results, Davies et al suggested that NC-derived progenitors in oral mucosa possess a multipotent capacity ${ }^{11)}$. Together, these results suggest that NCDCs in oral mucosa have roles in wound healing.

We also analyzed the potential of NC-derived hair follicle cells to differentiate into osteoblasts in response to bone morphogenetic protein (BMP)-2. Proliferative GFP-positive cells showed increased ALP activity and mineralization in the presence of BMP-2, suggesting that NC-derived hair follicle cells can differentiate into osteoblasts as a result of BMP-2 stimulation. Since abundant GFP-positive regions were seen in the buccal mucosa and hair follicle, we considered that this type of tissue would be beneficial for harvesting NCDCs for use in regenerative medicine strategies.

\section{Gene expression profile of neural crest-derived cells in adults}

NCDCs have been isolated from multiple sites within the body. Stemple and Anderson identified and isolated NCDCs from the trunk neural tube of rat embryos based on the expression of the low-affinity nerve growth factor receptor $(\mathrm{p} 75)^{31}$. Thereafter, NCDCs were isolated from the lamina propria of the oral mucosa by differential adhesion to fibronectin ${ }^{11)}$. In addition, a connexin-43-enriched human cell population isolated from periodontal ligament cells obtained from extracted third molars was shown to be multipotent in in vivo teratoma formation assays $^{32}$. Hauser et al recently reported a separation strategy based on magnetic cell sorting of p75-positive inferior turbinate cells, which formed larger neurospheres and proliferated faster than p75-negative inferior turbinate cells ${ }^{33)}$. Rat NCDCs were also isolated from the gut on embryonic day 14.5 by selecting p75 and $\alpha 4$ integrin double-positive fractions ${ }^{34)}$. Although p75 is useful as a marker for isolation of NCDCs, it is also expressed on other types of cells, and genetic lineage labeling techniques, such as $\mathrm{PO}$-Cre and Wnt1-Cre/EGFP, are not available for use with human samples. Therefore, it is very important to determine reliable cell surface molecules that are exclusively expressed on NCDCs found in adult human tissues. To examine whether GFPpositive cells in adult SMGs possess characteristic gene expression profiles, we compared gene expression patterns between GFP-positive and -negative cells using DNA microarray analysis, then selected genes related to cell surface molecules based on those findings ${ }^{24)}$. Compared to GFP-negative cells, GFP-positive cells expressed G protein-coupled receptor 4 and endothelin receptor type B (Ednrb) at higher levels, whereas Pdgfra and Pdgfrb were expressed at lower levels ${ }^{27)}$. Additionally, these two down-regulated genes (Pdgfra and Pdgfrb) are known to be important for salivary gland development ${ }^{26)}$.

Together, these results indicate that NCDCs in adult SMGs have characteristic gene expression profiles related to their cell surface molecules. In particular, it is known that Ednrb is required for migration of some NCDC derivatives, during which it interacts with Sox10, while Sox10 and the endothelin-3/EDNRB signaling pathway are required for normal enteric nervous system and melanocyte development ${ }^{35,36}$. We found that NCDCs isolated from adult SMGs had high expression levels of Sox10 and Ednrb, indicating that Sox10 and the endothelin-3/EDNRB signaling pathway contribute to the morphogenesis of SMGs, as well as the development of 
tissues derived from NCDCs other than those of the enteric nervous system and melanocytes. This study demonstrated the existence of NCDCs and a possible method for their isolation from adult SMGs, and our results suggest that some cell surface molecules might be identifiable as NCDC-specific markers in adult tissues.

In adults, NCDCs widely reside throughout the oral and maxillofacial regions, such as the palate, gingiva, tongue, and buccal mucosa, suggesting an important role. These tissues are also easily accessible for resection with a minimally invasive surgical procedure. NCDCs isolated from adult oral tissues have a stem cell phenotype, while they have also been shown to proliferate and differentiate into osteoblastic cells in vitro. Furthermore, cell sorting using a combination of specific cell surface marker genes may be an effective strategy to isolate NCDCs from various adult tissues with a high level of purity. We propose that NCDCs in oral tissues may be an ideal candidate cell source for regenerative medicine strategies.

\section{Acknowledgments}

We sincerely appreciate the help given by Professor Noriko Osumi (Department of Developmental Neuroscience, Tohoku University Graduate School of Medicine) in scientific discussions and for generously providing the P0-Cre/ $C A G$-CAT-EGFP mice. The results presented in this review are from investigations conducted by Dr. Miki Ono and Dr. Masahiro Takahashi (Assistant Professors, Department of Orthodontics, School of Dentistry, Showa University), and Dr. Eri Morisawa-Urano (Assistant Professor, Department of Prosthodontics, School of Dentistry, Showa University) during their studies as graduate students. We are deeply grateful to our colleagues in the Department of Biochemistry, School of Dentistry, Showa University, for their valuable insight and cooperation.

\section{Conflict of interest disclosure}

The authors have no conflict of interest to declare.

\section{References}

1) Gammill LS, Bronner-Fraser M. Neural crest specification: migrating into genomics. Nat Rev Neurosci. 2003;4:795805.

2) Basch ML, Bronner-Fraser M, García-Castro MI. Specification of the neural crest occurs during gastrulation and requires Pax7. Nature. 2006;441:218-222.

3) Duband JL, Monier F, Delannet M, et al. Epithelium-mesenchyme transition during neural crest development. Acta Anat (Basel). 1995;154:63-78.

4) Sieber-Blum M. Epidermal neural crest stem cells and their use in mouse models of spinal cord injury. Brain Res Bull. 2010;83:189-193.

5) Lumsden A, Sprawson N, Graham A. Segmental origin and migration of neural crest cells in the hindbrain region of the chick embryo. Development. 1991;113:1281-1291.

6) Osumi-Yamashita N, Ninomiya Y, Doi H, et al. The contribution of both forebrain and midbrain crest cells to the mesenchyme in the frontonasal mass of mouse embryos. Dev Biol. 1994;164:409-419.

7) Le Douarin NM, Kalcheim C. The Neural Crest. 2nd ed. New York: Cambridge University Press; 1999.

8) Chai Y, Jiang X, Ito Y, et al. Fate of the mammalian cranial neural crest during tooth and mandibular morphogenesis. Development. 2000;127:1671-1679.

9) Crane JF, Trainor PA. Neural crest stem and progenitor cells. Annu Rev Cell Dev Biol. 2006;22:267-286.

10) Delfino-Machin M, Chipperfield TR, Rodrigues FS, et al. The proliferating field of neural crest stem cells. Dev 
Dyn. 2007;236:3242-3254.

11) Davies LC, Locke M, Webb RD, et al. A multipotent neural crest-derived progenitor cell population is resident within the oral mucosa lamina propria. Stem Cells Dev. 2010;19:819-830.

12) Delorme B, Nivet E, Gaillard J, et al. The human nose harbors a niche of olfactory ectomesenchymal stem cells displaying neurogenic and osteogenic properties. Stem Cells Dev. 2010;19:853-866.

13) Ono M, Suzawa T, Takami M, et al. Identification and isolation of neural crest derived cells in nasal concha. Jpn J Tissue Cult Soc Dent Res. 2011;20:29-35.

14) Yoshida S, Shimmura $\mathrm{S}$, Nagoshi $\mathrm{N}$, et al. Isolation of multipotent neural crest-derived stem cells from the adult mouse cornea. Stem Cells. 2006;24:2714-2722.

15) Nagoshi N, Shibata S, Kubota Y, et al. Ontogeny and multipotency of neural crest-derived stem cells in mouse bone marrow, dorsal root ganglia, and whisker pad. Cell Stem Cell. 2008;2:392-403.

16) Suzuki J, Yoshizaki K, Kobayashi $\mathrm{T}$, et al. Neural crest-derived horizontal basal cells as tissue stem cells in the adult olfactory epithelium. Neurosci Res. 2013;75:112-120.

17) Yamauchi Y, Abe K, Mantani A, et al. A novel transgenic technique that allows specific marking of the neural crest cell lineage in mice. Dev Biol. 1999;212:191-203.

18) Kanakubo S, Nomura T, Yamamura K, et al. Abnormal migration and distribution of neural crest cells in Pax6 heterozygous mutant eye, a model for human eye diseases. Genes Cells. 2006;11:919-933.

19) Kawamoto S, Niwa H, Tashiro F, et al. A novel reporter mouse strain that expresses enhanced green fluorescent protein upon Cre-mediated recombination. FEBS Lett. 2000;470:263-268.

20) Kikuchi M, Hayashi R, Kanakubo S, et al. Neural crest-derived multipotent cells in the adult mouse iris stroma. Genes Cells. 2011;16:273-281.

21) Lemke G, Lamar E, Patterson J. Isolation and analysis of the gene encoding peripheral myelin protein zero. Neuron. 1988; 1:73-83.

22) Jiang X, Rowitch DH, Soriano P, et al. Fate of the mammalian cardiac neural crest. Development. 2000;127:16071616.

23) Ono M, Suzawa T, Takami M, et al. Localization and osteoblastic differentiation potential of neural crest-derived cells in oral tissues of adult mice. Biochem Biophys Res Commun. 2015;464:1209-1214.

24) Widera D, Zander C, Heidbreder M, et al. Adult palatum as a novel source of neural crest-related stem cells. Stem Cells. 2009;27:1899-1910.

25) Liu HX, Komatsu Y, Mishina Y, et al. Neural crest contribution to lingual mesenchyme, epithelium and developing taste papillae and taste buds. Dev Biol. 2012;368:294-303.

26) Yamamoto S, Fukumoto E, Yoshizaki K, et al. Platelet-derived growth factor receptor regulates salivary gland morphogenesis via fibroblast growth factor expression. J Biol Chem. 2008;283:23139-23149.

27) Takahashi M, Suzawa T, Yamada A, et al. Identification of gene expression profile of neural crest-derived cells isolated from submandibular glands of adult mice. Biochem Biophys Res Commun. 2014;446:481-486.

28) $\mathrm{Hu}$ YF, Gourab K, Wells C, et al. Epidermal neural crest stem cell (EPI-NCSC)--mediated recovery of sensory function in a mouse model of spinal cord injury. Stem Cell Rev. 2010;6:186-198.

29) Cooper JE, McCann CJ, Natarajan D, et al. In vivo transplantation of enteric neural crest cells into mouse gut; engraftment, functional integration and long-term safety. PLoS One (Internet). 2016;11:e0147989. (accesed 2016 Jan 29) Available from: http://dx.doi.org/10.1371/joumal.pone.0147989

30) $\mathrm{Xu} \mathrm{X}$, Chen $\mathrm{C}$, Akiyama K, et al. Gingivae contain neural-crest- and mesoderm-derived mesenchymal stem cells. $J$ Dent Res. 2013;92:825-832.

31) Stemple DL, Anderson DJ. Isolation of a stem cell for neurons and glia from the mammalian neural crest. Cell. 1992;71:973-985.

32) Pelaez D, Huang CY, Cheung HS. Isolation of pluripotent neural crest-derived stem cells from adult human tissues 
by connexin-43 enrichment. Stem Cells Dev. 2013;22:2906-2914.

33) Hauser S, Widera D, Qunneis F, et al. Isolation of novel multipotent neural crest-derived stem cells from adult human inferior turbinate. Stem Cells Dev. 2012;21:742-756.

34) Bixby S, Kruger GM, Mosher JT, et al. Cell-intrinsic differences between stem cells from different regions of the peripheral nervous system regulate the generation of neural diversity. Neuron. 2002;35:643-656.

35) Lee HO, Levorse JM, Shin MK. The endothelin receptor-B is required for the migration of neural crest-derived melanocyte and enteric neuron precursors. Dev Biol. 2003;259:162-175.

36) Stanchina L, Baral V, Robert F, et al. Interactions between Sox10, Edn3 and Ednrb during enteric nervous system and melanocyte development. Dev Biol. 2006;295:232-249.

[Received May 30, 2016 : Accepted July 1, 2016] 